\title{
Compact Low Power Wireless Gas Sensor Node With Thermo Compensation for Ubiquitous Deployment
}

\author{
Andrey Somov, Member, IEEE, Evgeny F. Karpov, Elena Karpova, Alexey Suchkov, Sergey Mironov, \\ Alexey Karelin, Alexander Baranov, Member, IEEE, and Denis Spirjakin
}

\begin{abstract}
Wireless sensor networks (WSNs) have recently been applied for industrial monitoring, including combustible and flammable gases monitoring. In this work, we present a wireless gas sensor node in which a widely used Wheatstone sensing circuit based on two sensors is exchanged with a single sensor circuit, as well as the associate gas measurement procedure. The core of the measurement procedure is the four-stage heating profile, which enables low power consumption of sensing circuit and thermo compensation adjustment. A thermo compensation algorithm is capable of avoiding the effect of the environmental temperature on the measurements by keeping stable zero-offset within $\pm 1 \mathrm{mV}$ and ensuring low absolute error within $0.1 \%$ vol. The thorough design of the sensor node allows it to fit into the $5.5 \mathrm{~cm}^{3}$ packaging, which ensures its true ubiquitous deployment in outdoor and industrial environment.
\end{abstract}

Index Terms-Advanced packaging, gas sensing, low power, power management, temperature compensation, wireless sensor network (WSN).

\section{INTRODUCTION}

A LMOST every industrial plant has some quantity of flammable and combustible liquids stored in its facility. Most of apartments and houses in Europe exploit methanebased $\left(\mathrm{CH}_{4}\right)$ boiler facilities to heat living spaces and use methane for cooking. Hydrogen, propane, butane, and methane are considered to be the ecological fuels for vehicles and are used at filling stations in many countries. Leaking of a combustible gas may lead to grave consequences, such as a house explosion with human victims (March 12, 2014, New York, USA) or gas poisoning. The detection of low gas concentration leaks is highly important in terms of ensuring the sanitary standards. Personnel working in gas mines and gas industry, in general, is subject to the occupational diseases. For example, factory workers dealing with methane have problems with the

Manuscript received October 10, 2014; revised December 28, 2014 and March 10, 2015; accepted April 07, 2015. Date of publication April 16, 2015; date of current version December 02, 2015. This work was supported by the Ministry of Education and Science of Russian Federation under Grant RFMEFI57714X0133. Paper no. TII-14-1173.

A. Somov is with CREATE-NET, 38123 Trento, Italy (e-mail: asomov@ create-net.org).

E. F. Karpov, S. Mironov, and A. Karelin are with the Scientific and Technical Center of Measuring Gas Sensors, 140004 Lyubertsy, Russia.

E. Karpova is with STANKIN, Moscow State Technological University, 127994 Moscow, Russia.

A. Suchkov, deceased, was with the Scientific and Technical Center of Measuring Gas Sensors, 140004 Lyubertsy, Russia.

A. Baranov and D. Spirjakin are with MATI, Russian State Technological University, 121552 Moscow, Russia (e-mail: baranov_64@mail.ru; denis. spirjakin@gmail.com).

Color versions of one or more of the figures in this paper are available online at http://ieeexplore.ieee.org.

Digital Object Identifier 10.1109/TII.2015.2423155 vegetative nervous system (hypotension and positive Aschner reflex).

Nowadays, a combustible gas leak at industrial facilities can be detected using two general technologies: electro catalytic (catalytic bead) and infrared (IR) absorption [1]. The detectors implemented by one of the technologies are deployed over a territory and wired in a network. This "wired" approach imposes a number of restrictions, especially for industrial premises:

1) large demand in terms of cable production;

2) dependency on a power grid;

3) time-consuming system deployment and debugging;

4) separation of the sensor wiring from the ac wiring.

Within the last years, the wireless sensor network (WSN) paradigm has been applied to a huge number of monitoring applications [2], [3] including gas monitoring. Indeed, there is a growing interest to gas WSN research [4]-[6] and deployment [7]. Until now, there are a number of widely used approaches for gas detection with WSN. The first solution relies on the energy-aware film (colorimetric) gas sensors [8], [9]. It is characterized by low power consumption (around $3 \mathrm{~mW}$ ) and long sensor response time (up to $5 \mathrm{~min}$ ) that fails to meet safety requirements [10]. In contrast, the gas WSN platforms employing laser spectroscopic trace-gas sensors [11] can detect and quantify numerous gas mixtures at part-per-million to part-per-billion (ppm-ppb) concentrations. Nonetheless, WSN platforms based on sensors of this type do not meet a long lifetime requirement consuming around $500 \mathrm{~mA}$. Similarly to spectroscopic approach, metal-oxide (MOX) and electrochemical [36] sensors operate properly in ppm concentrations and their application to the detection of combustible gas leakage in \% vol. concentrations will result in poisoning.

A viable tradeoff to secure both the long-term operation and sensor performance is the application of catalytic/ semiconductor sensors for combustible gas monitoring using WSN [12], [13]. However, semiconductor sensors have low selectivity to combustible gases such as methane, propane, and hydrogen, which makes the catalytic sensors most appropriate for this application. The principle of operation of the catalytic sensors is based on heating the sensing element and measuring its conductivity which changes in the presence of a gas in the atmosphere. A number of works report on the acceptable lifetime ( $>1$ year) and performance of WSN nodes with catalytic sensors [4], [14].

Since gas WSNs for hazardous gases detection are typically provided for deployment in boiler and industrial facilities, it implies some additional requirements and challenges [15]. Generic challenges, e.g., resource constraints [16], radio link 
quality [17], and WSN maintenance [18] have been extensively discussed in the literature so far. On the contrary, the application-specific challenges (such as the compact size of a gas sensor node to ensure true ubiquitous deployment and the gas measurement procedure guarantying both good sensitivity and low power consumption under significant temperature variation) are still subject for further research.

In this work, we present a WSN platform for combustible gases sensing with a special focus on industrial applications. The contribution of this work in terms of practical application is twofold.

1) Gas measurement procedure: We propose a four-stage heating profile which enables low power consumption of sensing circuit and a corresponding thermo compensation adjustment algorithm referred to below.

2) Thermo compensation adjustment: We propose and apply this algorithm during the measurement procedure to compensate for the ambient temperature change that helps avoiding the temperature impact on the measurements and, therefore, guarantying sensor stability and response.

This paper is structured as follows. The relevant works are reviewed in Section II. Section III presents the platform design, the resultant small-sized prototype, and its operation. The gas measurement procedure and thermo compensation algorithm are described in Section IV. The sensor node performance in terms of response and power consumption is evaluated in Section V. Finally, we provide concluding remarks in Section VI.

\section{RELATED WORKS}

WSN technology has opened up wide vista for environmental sensing and industrial applications including the hazardous gases detection. There are many examples of recently proposed gas WSN platforms aiming at long lifetime [4] and investigation of sensor performance [19]. Industrial applications, however, impose some additional requirements to WSN [15]. The sensor nodes for hazardous gases detection at plants, in some cases, have, e.g., to be deployed at various heights including the mixing emission height and difficult-to-access areas in indoor/outdoor conditions [20]. The industrial gas WSN platforms, therefore, must be of small scale factor to guarantee true ubiquitous WSN deployment and ought to ensure accurate measurements under the ambient temperature variation.

In this section, we survey the state-of-the-art literature that can be divided into three groups: 1) small form factor WSN platforms proposed or implemented for gas and similar monitoring applications; 2) thermo compensation algorithms and solutions for gas WSNs; and 3) power consumption of gas sensor nodes.

\section{A. Form Factor of WSN Nodes}

The first "classical" motes, e.g., MicaZ, Mica2, TelosB, supported basic sensing, processing, and wireless communications capabilities and were of the size of half a cigarette pack. Surprisingly enough, the size of the state-of-the-art commercial motes (FlyPort [21] and WaspMote [22]), supporting also the hazardous gases detection, is even bigger and thicker coming up to in the full cigarette pack size. These platforms, as a rule, are the modular devices attempting to meet the user's growing demands in data storage, multifunctionality, wireless communications, and emerging technologies, e.g., the Internet of Things [23].

The research gas WSN prototypes based on the catalytic/ semiconductor sensors are bulky devices [7], [13], [14], [24]. In fact, the commercial and certified catalytic gas sensors require large casing, e.g., TO-7, and power management circuits to enable their correct functioning. To ensure their long lifetime, the designers often equip the nodes with the D-type batteries [24] or provide separate power supplies for analogue (sensing) and digital (wireless communication and processing) circuits [14]. At the same time, WSN hardware platforms for other industrial applications, such as structural health monitoring [2] and ultraviolet flame detection [25], are of comparable size.

Miniaturization of sensing devices is a problem of top priority in the forthcoming era of Internet of Things [23] where tiny devices are ubiquitously deployed and seamlessly interconnected. To meet the small-scale factor requirement, CMOS/MEMS technologies [5] and modular approach [26] have become widely adopted in various industrial sectors. MEMS-based $3.9 \times 3.9 \times 3.5 \mathrm{~mm}$ sensor node presented in [28] is intended for temperature and humidity monitoring. A customized design supports signal processing, power management, and data transmission functions. Although the node has on-board integrated sensors, it can be easily interfaced with extra external digital/analogue sensors and energy harvesters, e.g., solar cells. PicoCube platform [26] is a $1 \mathrm{~cm}^{3}$ sensor node for tyre pressure monitoring supporting the modular and integrated approach. It includes five vertically stacked printed circuit boards (radio, power management, sensing, processing, and energy storage ones) packed in a plastic case. The long lifetime of the device is ensured by a harvester producing ac signal. The harvester is supposed to be an external though.

Yet, the size and power consumption of gas sensor nodes have been too large for the proper ubiquitous deployment. What we propose in our work is the significant reduction in the gas sensor node form factor achieved neither in the state-of-theart research prototypes nor in commercial hardware platforms [12]-[14], [21], [22]. This challenge is resolved by the combination of tiny electronic components in a modular way using the advanced casing.

\section{B. Temperature Compensation}

The ambient temperature varies significantly within a year and various industrial environments. This variation results in instability of sensor parameters and, therefore, in sensing accuracy [19]. In this section, we overview some relevant approaches proposed recently to overcome this problem.

The Wheatstone bridge is a commonly used sensing circuit in sensor nodes based on catalytic/semiconductor sensors [12], [24]. It includes two sensors, one active and one for reference. The active sensor is covered by a catalyst and is used to perform the measurements. The reference sensor is identical to the active one, but is not covered by a catalytic material. This makes the reference sensor insensitive to the gas concentration 
allowing the "automatic" compensation for environmental factors such as temperature and humidity. The Wheatstone circuit is very reliable in terms of compensation. However, it has two critical disadvantages in the scope of WSN: additional power consumption due to the application of two sensors and frequent "zero offset" calibrations, since the parameters of the sensors used vary unequally with respect to each other over time. To overcome these disadvantages, a sensing circuit with one sensor has been proposed recently [4], [29]. However, no temperature compensation techniques for the state-of-the-art gas WSN platforms based on one sensor can be found in the literature so far. This makes the state-of-the-art platforms practically useless for outdoor and industrial applications.

The approaches for temperature compensation proposed in [5] and [30] are modifications of the commonly used Wheatstone circuit. Okazaki et al. [30] propose a temperature compensation method for a sensing circuit employing active and reference catalytic sensors. The bottom line is that the sensing procedure requires the sensors being heated up to different temperatures with different heating currents: the active sensor is heated to above thermal decomposition temperature and the reference one to below thermal decomposition temperature. The authors claim that the method guarantees long-term stability, but lacks good selectivity, which limits its application.

The extra temperature sensor incorporated in a gas sensor to ensure the precise ambient temperature variation measurement is proposed in [5]. In contrast to the heater resistance measurement, this approach provides significant improvement of the gas sensor stability. However, integrating the temperature sensor has a number of drawbacks in terms of complexity and cost, as well as extra calibration for the temperature sensor apart from the gas one.

The novelty of our concept lies in using the temperature compensation approach for a single sensor-based sensing circuit. We propose the gas measurement procedure that applies the four-stage heating profile to a catalytic sensor. Measuring the sensor response after the stages, the software algorithm programmed in MCU compensates for ambient temperature. Our approach requires neither extra hardware components [5], nor the complicated generation of different heating currents [30].

\section{Power Consumption}

Nodes in WSN typically integrate four capabilities: computing, sensing, wireless communication, and power management for detecting a physical phenomena, for processing the data and forwarding it to a user over the network. Traditionally, wireless transceivers have been reported to be the most power-hungry device within a node [15]. Indeed, wireless transceivers typically consume up to 25 and $20 \mathrm{~mA}$ at $3.3 \mathrm{~V}$ in the transmit and receive modes, respectively. This is much higher than the power consumption of an $\mathrm{MCU}$, which requires up to $5 \mathrm{~mA}$ at $3 \mathrm{~V}$ in the active mode and just a few micro ampere in the sleep mode. Widely used sensors, such as a temperature, luminosity, and humidity ones, consume even less power than the MCU in the active mode. From the industrial perspective, there is a number of monitoring applications when a sensor requires a significant amount of power for correct functioning.
For example, the power consumption of catalytic and semiconductor gas sensors often exceeds that of a wireless transceiver [14]. We summarize the power consumption of widely used commercial and certified catalytic gas sensors manufactured by the world leading companies and compare their power consumption with other electronic components used on board in Table III. In this section, we focus on the power consumption of relevant gas sensor platforms as follows.

The power consumption of gas sensors may achieve 1-5 W, which prevents their application in WSNs. To address this problem, plenty of research efforts have been made recently [19]. In terms of power consumption of sensing circuit, this work improves the state-of-the-art on combustible and hazardous gases monitoring using WSN technology.

The early generic platform proposed by Wobscholl [34] consumes up to $1 \mathrm{~W}$, but allows for automatic sensor (catalytic/ semiconductor) calibration. Commercial sensing platforms for natural and LPG gases detection FlyPort [21] and WaspMote with the "Gases" extension sensing board [22], though having no automatic calibration option as compared with Wobscholl platform, are characterized by slightly reduced power consumption up to 800 and $280 \mathrm{~mW}$, respectively. The advantage of commercial platforms is the simplified application design available by the code reuse and technical support, as well as the minimum time required for platform debugging. These platforms, however, ignore the influence of environmental conditions resulting in inaccurate measurements.

Platforms for methane monitoring proposed in [12] and [24] largely improve on power consumption in spite of using Wheatstone sensing circuit based on two sensors: catalytic and semiconductor, respectively. The key advantage of this circuit is the "automatic" compensation for the ambient conditions, since the resultant circuit response is calculated on the basis of responses from an active (with catalyst) and reference (without catalyst) sensors. The sensor platform proposed in [24] consumes $264 \mathrm{~mW}$ and uses the pulsed heating profile instead of continuous sensor heating [21], [22], [34]. This approach, however, can damage the sensor due to frequent ON/OFF of heating pulses. Another drawback of the pulsed approach is that the moisture does not fully evaporate between the pulses, thus leading to inaccurate measurements. The continuous heating profile used in [12] addresses the problem of the incomplete moisture evaporation and, due to careful design and power management, improves the power consumption up to $227 \mathrm{~mW}$. Yokosawa et al. [13] present a similar sensor node for hydrogen leak monitoring at petrol stations. This node has 200-mW power consumption and long sensor response. Similar research on the investigation of heating profiles has been done for MOX sensors, which are applied for hazardous gases detection in ppm range [35], [36]. These works make an emphasis on the reduction in sensor power consumption by finding a tradeoff among the number of pulses forming the burst, the pulse width, and sleep time between pulses. The lifetime of a battery is 30 times improved, though temperature and humidity noticeably affect the resultant response.

The sensor nodes presented in [14] and [4] are based on single catalytic sensor sensing circuit and have the lowest power consumption 124.30 and $85.68 \mathrm{~mW}$, respectively. Both works 


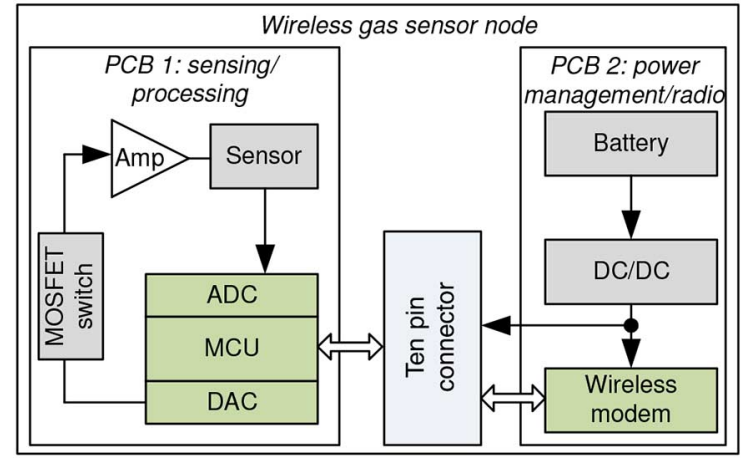

Fig. 1. Block diagram of the wireless gas sensor node.

focus on reducing power consumption and compare the single sensor approach with the Wheatstone circuit. The comparative study shows that the single sensor circuit is approximately $65 \%$ more energy efficient. Focusing on the energy-aware design, these works do not consider the change in environmental temperature which results in inaccurate measurements. The heating profiles designed in [4] and [14] are aimed at 1) power consumption reduction and 2) moisture evaporation. The heating (sub)pulses are designed in the way that does not allow their application for temperature compensation. Our work performs temperature compensation and ensures the low average power consumption of $67 \mathrm{~mW}$ of sensing circuit. This is achieved by the improved hardware together with power management and a heating profile designed for compensation adjustment. The heating profile used in this work allows for the increase in catalytic sensor stability in the ambient environment.

\section{Platform Design}

The proposed hardware platform shown in Fig. 1 comprises two $3 \mathrm{~cm}^{2}$ PCBs connected by a standard commercial ten-pin connector. The platform includes four units: the processing and sensing units are realized by $P C B \quad 1$; wireless communication and power conditioning ones are provided by realized by $P C B 2$. All electronic components incorporated in the units are to meet the following requirements: energy efficiency, small size, compatibility of the components, and their simple interface.

The processing unit is implemented around the ADuC 845 Microcontroller (MCU). The selection of the MCU was mainly driven by the following requirements: low power consumption, on-chip temperature sensor, and precise analog-to-digital converter (ADC) and digital-to-analog converter (DAC) integrated in MCU. Both devices must be as precise as possible to generate the sensor's heating profile, to make intermediate calculations (see Section III-B), to calculate the output signal in digital form and send it to the wireless communication unit. The 24-bit ADC performs signal processing without extra signal amplifiers that generate noise [4], while the 12-bit DAC generates the four-stage dynamic heating profile for the sensor.

The sensing unit is built on the one-sensor-based sensing circuit (voltage divider) [4]. The sensing circuit is disabled by a MOSFET switch when it does not conduct the sensing of the
TABLE I

Summary of DTK-3 Sensor Parameters

\begin{tabular}{ll}
\hline \hline \multicolumn{1}{c}{ Parameter } & \multicolumn{1}{c}{ Value } \\
\hline Max. power consumption of one sensing element (SE) & $75 \mathrm{~mW}$ \\
Resistance of one SE in normal conditions & $10-14 \Omega$ \\
Effective range of methane concentration & $0-5 \%$ vol. \\
Level of explosion isolation & OexiasIIBT6 \\
Output response setup time & $<5 \mathrm{~s}$ \\
\hline \hline
\end{tabular}

atmosphere. An operational amplifier (Amp) ensures the sufficient level of heating current for the sensor. A more detailed analysis of the sensing circuit is presented in Section IV-A. We use the catalytic sensor DTK-3 manufactured by NTC IGD, Russia. The sensor height is $9.5 \mathrm{~mm}$ and diameter $9 \mathrm{~mm}$; power consumption is $75 \mathrm{~mW}$ in the continuous measurement mode and full duty cycle. Its low power consumption is achieved by applying a heater implemented as $10 \mu \mathrm{m}$ platinum microwire in glass insulation. Table I summarizes key parameters of DTK-3 sensor. We note that the state-of-the-art catalytic sensors employ the $20 \mu \mathrm{m}$ heaters and are of $10 \mathrm{~mm}$ height and $12 \mathrm{~mm}$ diameter with the power consumption of $150-600 \mathrm{~mW}$ [24]. The sensor power consumption in the proposed design can further be reduced by using the dynamic heating profile and decreasing the duty cycle for the sensing task. For our purposes, we use one sensor-based sensing circuit in contrast to the widely used Wheatstone sensing circuit employing two sensors [12].

The wireless communication unit employs the low power ETRX3 wireless modem supporting IEEE 802.15.4 standard (ZigBee specification) and transmitting in unlicensed $2.4 \mathrm{GHz}$ ISM band. The modem has an integrated chip antenna used in this design (up to $25 \mathrm{~m}$ ) and a connector for an external antenna to enable a boost mode allowing data transmission for up to $350 \mathrm{~m}$. Besides that, the modem has a number of self-x features enabling, for instance, WSN self-configuration and self-diagnostics which significantly reduce WSN debugging and deployment time.

The power conditioning unit provides the stable $2.7 \mathrm{~V}$ voltage supply for the sensor node. The core element of the unit is a TPS61200 dc/dc in a tiny $3 \times 3 \mathrm{~mm}$ package. The device generates stable output voltage from 0.3 to $5.5 \mathrm{~V}$ on its input. We use as a power supply the button type Li battery CR-2477 (3 V, $1000 \mathrm{mAh}$ ) for experimenting and proof of concept reasons. Since the objective is ensuring low power consumption rather than long lifetime, we leave the user an opportunity to choose any battery that meets the node's technical and application lifetime requirements.

The sensor node prototype is shown in Fig. 2. To meet the small form factor requirement for the sensor node, we consider an approach, where tiny electronic components are combined in a modular fashion using advanced A3 packaging by Alphasense, while maintaining energy efficiency and integration density.

\section{A. Sensor Node Operation}

Let us consider a WSN consisting of wireless gas sensor nodes, wireless actuators, and gateway. The WSN topology 


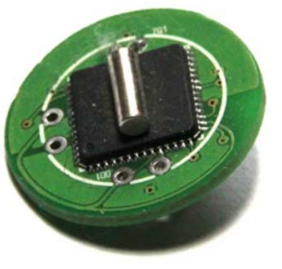

(a)

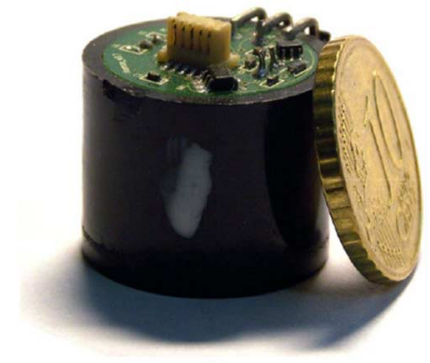

(b)
Fig. 2. Sensor node: (a) $3 \mathrm{~cm}^{2} \mathrm{PCB}$ with $\mathrm{MCU}$ (top view) and sensor; and (b) final $5.5 \mathrm{~cm}^{3}$ prototype compared to 10 Euro cent coin.

is of star configuration. The WSN gateway sets up the network parameters automatically. The sensor nodes are the energy constrained devices performing gas sensing and communicating with actuators and gateway via the wireless channel using the IEEE802.15.4 standard and the low power wireless specification ZigBee. To guarantee energy saving, the sensor nodes spend most of their time in the sleep mode and perform their sensing task once a minute and their communication task for synchronization and service purposes every 5 min unless a dangerous gas concentration has been detected. The actuators periodically communicate with the sensor nodes and gateway to be in time for closing a gas valve in a dangerous situation. The gateway connects the actual gas WSN with the real world, i.e., the WSN operator and/or gas service crew.

If the sensor node detects $\leq 0.5 \%$ vol. gas concentration, it sends an alarm message to the gateway which decides what to do next. When the measured gas concentration is between 0.5 and $1 \%$ vol., the sensor node immediately sends a request to the actuator to close the gas valve and, in parallel, notifies the WSN operator about the dangerous situation via the gateway. The methane lower explosive limit (LEL) is 5\% vol.

\section{Measurement Procedure}

In this section, we present the sensing circuit and then propose and discuss the dynamic heating profile enabling the ambient temperature compensation algorithm for the measurement procedure.

A similar approach has been presented in [4], [14], and [24]. The authors propose a straightforward approach for the sensor response measurement by applying frequent turning ON/OFF heating pulses in [24]. This technique helps to reduce the power consumption, but has a number of disadvantages: moisture does not fully evaporate from the sensor, which results in inaccurate measurements and the sensors can be damaged in a short period of time due to frequent and drastic heating pulses variations. Both works [4], [14] focus on how to reduce power consumption. Being concentrated on the energy-aware design, the authors do not consider the change in environmental temperature which leads to inaccuracy of measurements. The heating profiles designed in [4] and [14] are aimed at power consumption reduction and moisture evaporation from the sensor surface. Our solution, on the contrary, enables the temperature compensation to increase the sensor stability and response in

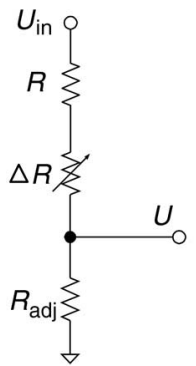

(a)

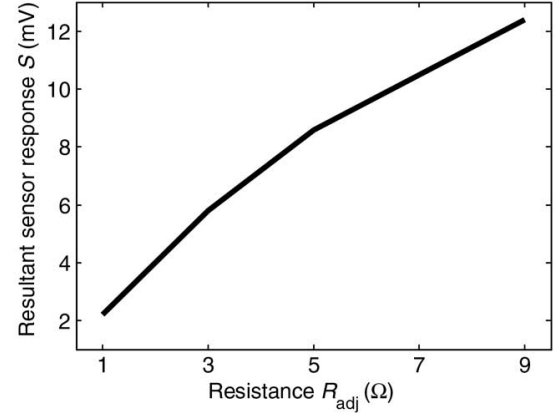

(b)
Fig. 3. (a) Sensing circuit based on one sensor shown as $R$ with its changing resistance for $\Delta R$ during sensing and (b) an example of sensor resultant response $S$ in the presence of $2 \%$ vol. methane concentration depending on $R_{\text {adj }}$ resistor value in the sensing circuit.

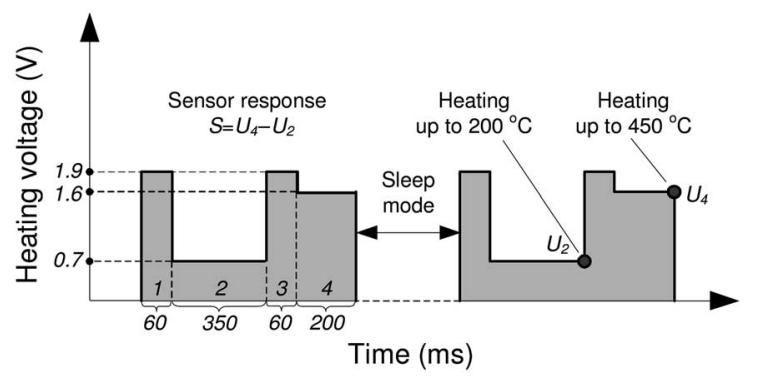

Fig. 4. Four-stage dynamic heating profile.

the ambient environment and ensures the low average power consumption of the sensor node.

\section{A. Sensing Circuit}

In this work, we use one-sensor-based sensing circuit (divider) [4] employing the catalytic sensor. The sensors of this type are typically used in the LEL gas concentration range, i.e., for detecting combustible gases, due to their high sensitivity, selectivity, linear, and fast response.

Fig. 3(a) shows the sensing circuit where the catalytic sensor represented as its resistance in normal conditions $R$ is embedded in one arm of the voltage divider circuit. The operating principle of a catalytic sensor provides for its heating up to $450{ }^{\circ} \mathrm{C}$ and measuring its change in resistance $\Delta R$. Resistance $R_{\text {adj }}$ is the predefined and precise resistor needed to calculate the divider parameters, e.g., heating current. The exact value of $R_{\text {adj }}$ should be chosen with care: by increasing $R_{\text {adj }}$ the user improves the circuit response [see Fig. 3(b)] and at the same time increases the power consumption $\left(P_{\text {adj }}=U_{\text {in }}^{2} \cdot R_{\text {adj }}\right)$.

Fig. 3(b) shows how sensitivity depends on $R_{\text {adj }}$ in the active mode. The sensor temperature during sensing is $450{ }^{\circ} \mathrm{C}$.

The voltage divider sensing circuit is more energy efficient than the Wheatstone one due to application of one sensor. However, it has an important disadvantage being unable to compensate for the effect of ambient temperature as compared with the Wheatstone circuit where the resultant response from the active and reference sensors provides this compensation "by default" [4]. In the following sections, we demonstrate how to address this problem. 


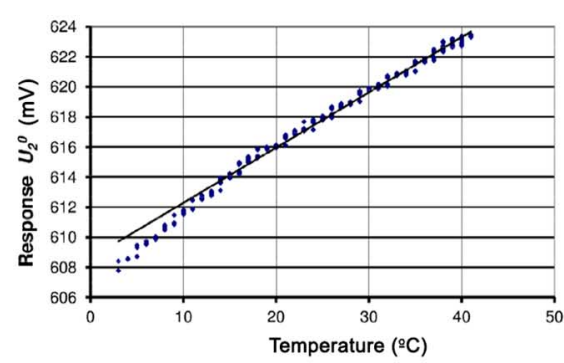

(a)

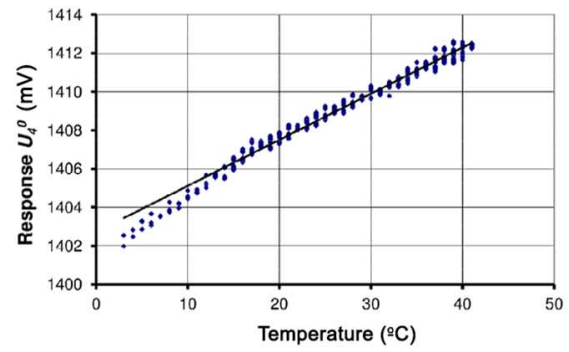

(b)

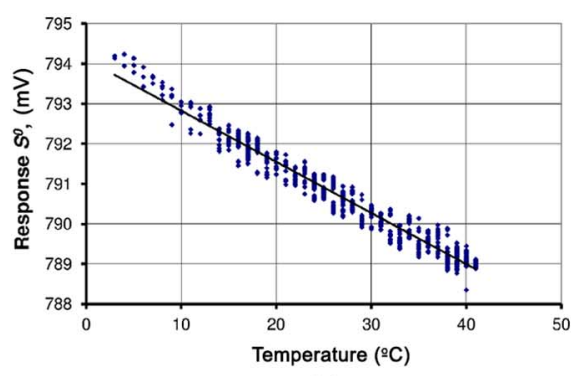

(c)

Fig. 5. Sensor response (a) $U_{2}^{0}$, (b) $U_{4}^{0}$, and (c) resultant output response $S^{0}$ under the environmental temperature change for $38^{\circ} \mathrm{C}$ at $0 \%$ gas concentration in the atmosphere where solid line is approximation and dots are experimental values.

\section{B. Heating Profile}

The dynamic heating profile is shown in Fig. 4. The profile includes four stages: two heating and two measuring ones. The first stage ensures the sensor preheating and lasts for $60 \mathrm{~ms}$ at $1.9 \mathrm{~V}$. During the second stage the moisture evaporation (without gas burning) occurs. At the end of this stage lasting $350 \mathrm{~ms}$ at $0.7 \mathrm{~V}$, the sensor temperature is stabilized at $200^{\circ} \mathrm{C}$ and system measures sensor response $U_{2}$, a "reference measurement," showing how the environment conditions (without burning) have affected the measurement. Basically, this measurement has the same function as the reference sensor in the Wheatstone sensing circuit [24]. The objective of the third stage which lasts for $60 \mathrm{~ms}$ at $1.9 \mathrm{~V}$ is to heat the sensor to higher temperature when methane oxidation begins.

At the fourth stage lasting for $200 \mathrm{~ms}$ at $1.6 \mathrm{~V}$, the sensor operates as the active one in the Wheatstone sensing circuit.

At the end of this stage, the temperature is stabilized at $450{ }^{\circ} \mathrm{C}$ and system measures the sensor response $U_{4}$. The temperature of the sensing layer is proportional to methane concentration in the environment. When the measurement is conducted, the sensor goes to the sleep mode. It is worth noting that the average voltage during the measurement procedure $(0.67 \mathrm{~s})$ is around $1.2 \mathrm{~V}$. For the Wheatstone circuit, this value is $2.8 \mathrm{~V}$ [4].

The sensor output signal $S$ in $\mathrm{mV}$ is proportional to a gas concentration in the atmosphere and is calculated as follows:

$$
S=\left(U_{4}-U_{2}\right)-S^{0}
$$

where

$$
S^{0}=U_{4}^{0}-U_{2}^{0}
$$

where $S^{0}$ is the sensor output signal in $\mathrm{mV}$ at $0 \% \mathrm{CH}_{4}$ concentration in the atmosphere, $U_{2}^{0}$ and $U_{4}^{0}$ are the sensor responses in $\mathrm{mV}$ at $0 \% \mathrm{CH}_{4}$ concentration in the atmosphere after second and fourth stages, respectively.

Replacing the Wheatstone sensing approach by the single sensor one results in the appearance of an excess error. Its nature lies in the different heat loss at various heating temperatures (see Fig. 4).

Fig. 5 demonstrates the experimentally derived curves of the sensor response at different measurement stages $\left(U_{2}^{0}, U_{4}^{0}\right.$, and $S^{0}$ ) with respect to the environmental temperature change for $38^{\circ} \mathrm{C}$. The response change is 15 and $10 \mathrm{mV}$ for $U_{2}^{0}$ and $U_{4}^{0}$ signals, respectively. The maximum drift of the zero offset for this sensing circuit is $0.33 \%$ vol. instead of $0.1 \%$ vol. tolerance at the temperature change for $38^{\circ} \mathrm{C}$. The obtained error is of additive character and will entail an equal error during methane detection of no $0 \%$ vol. concentration.

\section{Thermo Compensation Adjustment Algorithm}

To avoid an excess error during the measurement procedure and under the changing environmental temperature, we propose an algorithm for measurements' correction.

At the time of sensor calibration in normal conditions described in standard GOST IEC 6007929.1, we measure the current $I_{2}^{0}$ and $I_{4}^{0}$ flowing through the sensor during the second and fourth heating stages using $R_{\text {adj }}$ as a shunt and calculate sensor resistance $R_{2}^{0}$ and $R_{4}^{0}$, respectively,

$$
\begin{aligned}
& R_{2}^{0}=U_{2}^{0} / I_{2}^{0} \\
& R_{4}^{0}=U_{4}^{0} / I_{4}^{0} .
\end{aligned}
$$

In real conditions, the system measures the respective sensor responses $U_{2}$ and $U_{4}$ allowing for the fact that the heating current in normal and real conditions is equal $\left(I_{2}=I_{2}^{0}\right.$ and $\left.I_{4}=I_{4}^{0}\right)$ and calculates the sensor respective resistances $R_{2}$ and $R_{4}$ using (3) and (4), respectively.

The system then calculates, using (5), the sensor resistance change at the second heating stage $\Delta R_{2}$, which is only influenced by the environmental temperature change (not by methane burning). Using (6), the system calculates the sensor resistance change at the fourth stage $\Delta R_{4}$, which is influenced by both the environmental temperature change and methane burning

$$
\begin{aligned}
& \Delta R_{2}=\left(R_{2}-R_{2}^{0}\right) \\
& \Delta R_{4}=\left(R_{4}-R_{4}^{0}\right) .
\end{aligned}
$$

The corrected sensor responses $U_{2 C}$ and $U_{4 C}$ at the second and fourth heating stages, respectively, are calculated as follows:

$$
\begin{aligned}
& U_{2 C}=U_{2}-\Delta R_{2} \cdot I_{2}=U_{2}-\left(R_{2}-R_{2}^{0}\right) \cdot I_{2} \\
& U_{4 C}=U_{4}-\Delta R_{4} \cdot I_{4}=U_{4}-\left(R_{4}-R_{4}^{0}\right) \cdot I_{4} .
\end{aligned}
$$

The resultant sensor response is proportional to methane concentration in the atmosphere and is calculated using (9)

$$
S_{C}=U_{4 C}-U_{2 C} .
$$




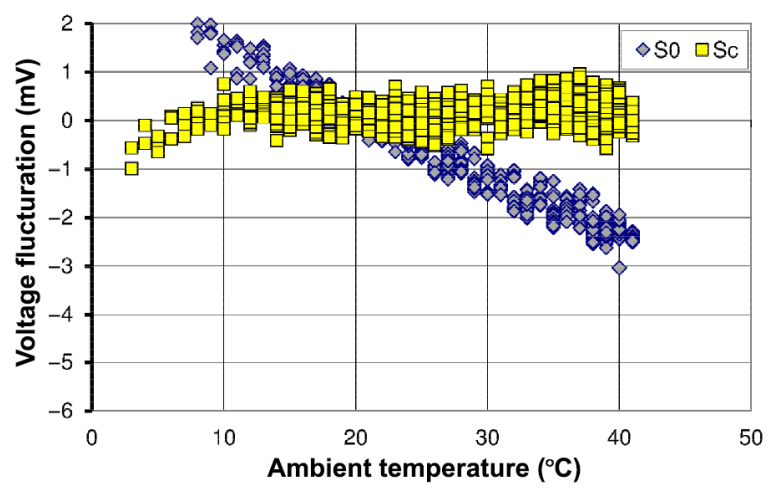

Fig. 6. Sensor output voltage fluctuation with thermo compensation $\left(S_{C}\right)$ and without thermo compensation $\left(S^{0}\right)$ adjustment at $0 \%$ vol. methane concentration and ambient temperature change for $38^{\circ} \mathrm{C}$.

The sensor calibration in gas environment is conducted with the adjusted sensor response signals calculated using (7)-(9), which helps avoiding an excess error. We note that sensor calibration is required once a year according to standard GOST IEC 6007929.1.

\section{Evaluation AND Discussion}

In this section, the proposed temperature compensation algorithm is experimentally assessed in terms of sensor response at varying ambient temperature and gas concentration in the atmosphere. In particular, we evaluate the sensor zero-offset, absolute measurement error, and sensor response. Finally, an estimation of the sensor node power consumption and life time is given. In all experiments, we apply to the sensor the heating profile described in Section IV-B.

\section{A. Response}

To evaluate the sensor response, we conduct three experiments. The goal of the first experiment is to evaluate the zero offset with and without the application of thermo compensation adjustment at the ambient temperature change. To conduct the experiment, we put the sensor node in a temperature chamber. The MCU registers both temperature values with offset drift $10 \mathrm{nV} /{ }^{\circ} \mathrm{C}$ and the sensor output voltage using ADC with $\pm 10 \mu \mathrm{V}$ error. The results of the first experiment are demonstrated in Fig. 6. It shows the voltage fluctuation of zero offset caused by the environmental temperature change in the absence of methane. In the best case, the voltage fluctuation must be $0 \mathrm{mV}$ so as not to affect the sensor response in the presence of the gas (see Section IV-B). Without applying the thermo compensation adjustment, this requirement is only achieved at normal conditions at the time of sensor calibration, i.e., at $20^{\circ} \mathrm{C}$. The experimental results show that the application of temperature compensation algorithm reduces the voltage fluctuation and guarantees the sensor response stability, i.e., the error of the adjusted resultant sensor response $S_{c}$ at the ambient temperature change for $38^{\circ} \mathrm{C}$ does not exceed $2 \mathrm{mV}$ at $0 \%$ vol. methane concentration. The absolute error does not exceed $0.1 \%$ vol. (equal to $\pm 2 \mathrm{mV}$ ) defined as a threshold by
TABLE II

Absolute Measurement Error (\% vol.) of Test Gas Mixture $\mathrm{CH}_{4}$ With (w) AND Without (w/O) The Application of Thermo COMPENSATION

\begin{tabular}{|c|c|c|c|c|c|c|}
\hline \multirow{2}{*}{$\mathrm{T}\left({ }^{\circ} \mathrm{C}\right)$} & \multicolumn{2}{|c|}{$\mathrm{CH}_{4} 0.5 \%$ vol. } & \multicolumn{2}{|c|}{$\mathrm{CH}_{4} 1 \%$ vol. } & \multicolumn{2}{|c|}{$\mathrm{CH}_{4} 2.5 \%$ vol. } \\
\hline & $\mathrm{w}$ & w/o & $\mathrm{w}$ & w/o & $\mathrm{W}$ & w/o \\
\hline 3 & 0.04 & 0.14 & 0.04 & 0.14 & 0.05 & 0.15 \\
\hline 20 & 0.01 & 0.0002 & 0.02 & 0.0001 & 0.01 & 0.0002 \\
\hline 41 & 0.04 & 0.12 & 0.02 & 0.13 & 0.02 & 0.13 \\
\hline
\end{tabular}

the standards [10], [20] and is $0.05 \%$ in the worst case, e.g., at $3{ }^{\circ} \mathrm{C}$ and $36^{\circ} \mathrm{C}$. The error of $S^{0}$ response is $5 \mathrm{mV}$, the absolute error grows with the temperature change from $20^{\circ} \mathrm{C}$, e.g., below $7{ }^{\circ} \mathrm{C}$ and above $33^{\circ} \mathrm{C}$ the absolute error exceeds $0.1 \%$ vol. This experiment demonstrates that the application of the proposed algorithm helps keeping the zero-offset properly to meet the absolute error requirement.

The goal of the second experiment is to evaluate the absolute measurement error in the presence of gas and at different temperatures. Table II demonstrates the absolute measurement error at $0.5,1$, and $2.5 \%$ vol. $\mathrm{CH}_{4}$ test gas mixture concentrations at three temperatures. Each experiment was conducted 50 times on 10 identical sensors with and without the application of thermo compensation adjustment. As in previous experiment, the sensor is calibrated at $20^{\circ} \mathrm{C}$, which results in precise measurements for the sensor without the thermo compensation adjustment. However, if the ambient temperature varies from the calibration temperature, the absolute error grows and does not meet the requirement of $0.1 \%$ vol. absolute error. In contrast, the application of thermo compensation adjustment keeps the absolute error stable enough during the temperature change and in none of the cases it exceeds $0.1 \%$ vol. absolute error.

The proposed approach helps to simplify the WSN maintenance. There are two general approaches to ensure the proper sensors operation at the ambient temperature: 1) sensors calibration in all range of operating temperatures and recording these values in the memory of $\mathrm{MCU}$; and 2) compensation for the ambient temperature proposed in this work. This first approach is a time consuming one especially bearing in mind that a WSN consists of high number of nodes, which have to operate autonomously as long as possible. The algorithm proposed in this work ensures high accuracy and is programmed in the memory of MCU. It is processed immediately together with sensing.

We note here that the proposed algorithm can be implemented on any catalytic sensors. However, we do advise to carefully check the maximum heating ratings of other sensors so as not to damage them.

Apart from ensuring the measurements' stability, the thermo compensation algorithm improves the sensor response. Fig. 7 shows the sensor response with and without the thermo compensation algorithm at five methane concentrations. The "etalon" reference sensor response signal is $30 \mathrm{mV}$ at $1 \%$ vol. in Wheatstone circuit [4]. This chart shows that our approach approximately two times improves the state-of-the art results, i.e., sensing circuits based on one sensor. The Wheatstone circuit has better response, but is $65 \%$ more power "hungry" than the one sensor circuit (see Section II-C). The energy efficiency 


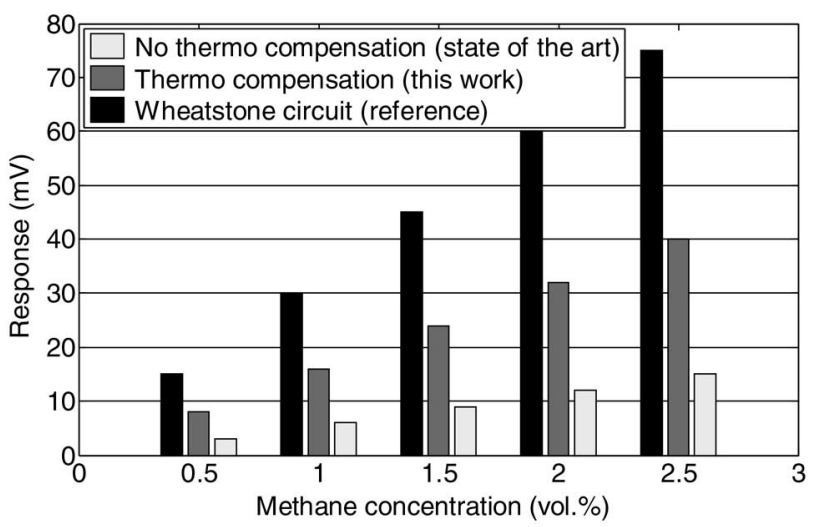

Fig. 7. Sensor response to $0.5,1,1.5,2$, and $2.5 \%$ vol. of methane with and without the application of thermo compensation adjustment algorithm.

TABLE III

Average Power Consumption of the Gas Sensor Node ELECTRONIC COMPONENTS: COMPARATIVE STUDY

\begin{tabular}{|c|c|c|}
\hline \multirow{2}{*}{ Device } & \multicolumn{2}{|c|}{ Power consumption (mW) } \\
\hline & $\begin{array}{l}\text { Active } \\
\text { mode }\end{array}$ & $\begin{array}{l}\text { Sleep } \\
\text { mode }\end{array}$ \\
\hline${ }^{*}$ Catalytic gas sensor DTK-3, NTC IGD & & \\
\hline Continuous heating (Wh. circuit) & 150 (at $2.8 \mathrm{~V}$ ) & 0 \\
\hline Dynamic heating (this work) & 67 & 0 \\
\hline${ }^{*}$ Radio (TX) ETRX3, Telegesis & $84($ at $2.7 \mathrm{~V}$ ) & 0.003 \\
\hline " Radio (RX) ETRX3, Telegesis & 76 (at $2.7 \mathrm{~V}$ ) & 0.003 \\
\hline "MCU ADuC845, Analog Devices & $11($ at $2.7 \mathrm{~V})$ & 0.066 \\
\hline Luminosity sensor TSL2561, Adafruit & 1.5 & 0 \\
\hline Humidity sensor $808 \mathrm{H} 5 \mathrm{~V} 5$, Sensera & 1.25 & 0 \\
\hline $\begin{array}{l}\text { Temperature sensor TMP102, Texas } \\
\text { Instruments }\end{array}$ & $33 \mu \mathrm{W}$ & 0 \\
\hline Catalytic gas sensor TGS2610, Figaro & 280 & 0 \\
\hline Catalytic gas sensor NAP-66A, Nemoto & 360 & 0 \\
\hline $\begin{array}{l}\text { Catalytic gas sensors MC series, } \\
\text { Huanwei }\end{array}$ & up to 600 & 0 \\
\hline
\end{tabular}

* Components used in this work.

of the one sensor-based approach can be explained by exploiting just one sensor in the sensing circuit and the application of four stage heating profile instead of continuous one. In fact, the proposed approach generates enough response signal for its processing in the MCU and does not require complicated filtering with extra hardware components [4].

\section{B. Power Consumption and Life Time Estimation}

In this section, we experimentally evaluate the power consumption of the sensing circuit and estimate the lifetime of the gas sensor node. Table III demonstrates the node performance in terms of power consumption in the active and sleep modes. Besides, we note that we focus on experimental evaluation of sensor current consumption since it is a core element for the heating profile and thermo compensation algorithm and is the most power hungry component of the node. At the same time, the performance of the MCU and wireless modem is studied in their specifications and relevant papers [14].

Fig. 8 shows the current consumption of the sensing circuit during the gas measurement procedure which takes $0.67 \mathrm{~s}$. This current flows through the precise resistor $R_{\text {adj }}$ shown

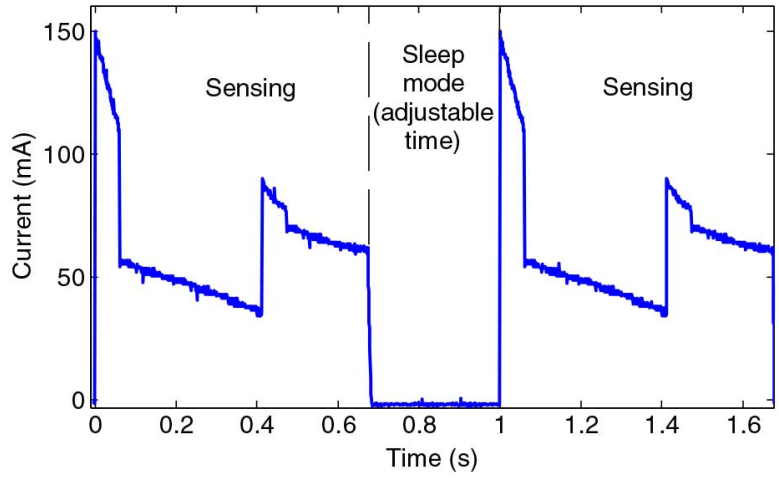

Fig. 8. Current consumption diagram of the sensing circuit.

in Fig. 3(a) and its average value is $56 \mathrm{~mA}$. The power consumption is approximately $67 \mathrm{~mW}(56 \mathrm{~mA} \times 1.2 \mathrm{~V}$, see Section IV-B). We note that the same sensor consumes $150 \mathrm{~mW}$ in the Wheatstone circuit $(75 \mathrm{~mW} \times 2=150 \mathrm{~mW})$ in the continuous measurement mode. However, to effectuate the measurement of the total power consumption of the sensor node is a non trivial task due to the application of dc/dc which inherently operates in the pulse mode and performs the circuit break between the power supply and itself for a short period of time. We, consequently, estimate the power consumption of MCU and the wireless transceiver operating at $2.7 \mathrm{~V}$ (the power consumption of other electronic components is negligible). MCU is in active mode during the measurement procedure and wireless communication and its maximum power consumption is about $11 \mathrm{~mW}$. Our empirical study has shown that the wireless modem operated for $0.11 \mathrm{~s}$ to transmit an alarm message and receive an acknowledgement [4]. The average power consumption of the transceiver in the case of one transmission per minute will be about $0.15 \mathrm{~mW}(84 \mathrm{~mW}+76 \mathrm{~mW}) / 2 *(0.11 \mathrm{~s} / 60 \mathrm{~s})$, which is negligible as compared to other components. If the measurement occurs one time per minute, the total node consumption is about $1.04 \mathrm{~mW}$. The conditions are: the measurements cycle is $0.67 \mathrm{~s}, \mathrm{MCU}$ is in active mode during the measurement cycle $(0.67 \mathrm{~s})$ and data transmission $(0.11 \mathrm{~s})$, the transceiver is in the transmission/receiving mode $(0.11 \mathrm{~s})$. The total power consumption of the sensor node is approximately $161 \mathrm{~mW}$ for the Wheatstone sensing circuit and continuous measurement procedure and sensor heating, $78 \mathrm{~mW}$ for the voltage divider sensing circuit with continuous measurement procedure and dynamic sensor heating, and $1.04 \mathrm{~mW}$ for the voltage divider sensing circuit with one time per minute periodic measurement procedure and dynamic sensor heating. The results are summarized in Table IV.

This estimation helps us evaluate the lifetime of the gas sensor node. The sensor node lifetime is assessed for two cases taking into account the principles of the sensor node operation described in Section III-A. If the sensor node conducts measurements once a minute and there are no detections of dangerous gas concentration and, therefore, the wireless modem is kept in the sleep mode. In this case, the sensor node can operate for about 3370 h (140 days) with CR-2477 battery (see Section III). In the case of data transmission one time per minute, the node can operate for about $2880 \mathrm{~h}$ (120 days). 
TABLE IV

Average Power Consumption of the Gas Sensor Node ELECTRONIC COMPONENTS IN DIFFERENT MEASUREMENT Modes

\begin{tabular}{|c|c|c|c|}
\hline \multirow[b]{2}{*}{ Device } & \multicolumn{3}{|c|}{ Power consumption (mW) } \\
\hline & $\begin{array}{c}\text { Continuous } \\
\text { mode, } \\
\text { Wh. circuit } \\
\text { (state-of-the-art) }\end{array}$ & $\begin{array}{c}\text { Continuous } \\
\text { mode, voltage } \\
\text { div. circuit (this } \\
\text { work) }\end{array}$ & $\begin{array}{l}\text { Periodic (every } \\
60 \text { s), voltage div. } \\
\text { circuit (this } \\
\text { work) }\end{array}$ \\
\hline $\begin{array}{l}\text { Sensing } \\
\text { circuit }\end{array}$ & 150 & 67 & 0.75 \\
\hline MCU & 11 & 11 & 0.14 \\
\hline $\begin{array}{l}\text { Radio } \\
\text { (every } 60 \mathrm{~s} \text { ) }\end{array}$ & 0.15 & 0.15 & 0.15 \\
\hline Total & 161 & 78 & 1.04 \\
\hline
\end{tabular}

The lifetime of the sensor node can be prolonged by applying a battery with the improved capacity or by wiring the batteries in parallel. The most important result is the low average power consumption which, together with the platform small scale factor and the temperature compensation, opens up wide vista for outdoor and industrial monitoring applications.

It should be noted here that lifetime of the sensor nodes for the combustible gases application can be improved through employing the energy harvesting technology and advanced energy management [27]. Due to the power hungry nature of this application, a hybrid energy harvesting approach may be suggested as more efficient one [2]. Extra energy savings can be achieved through various energy aware techniques, e.g., by providing the proper packaging insulation of the methane sensor [31], by performing the careful analysis of the sensor node power consumption using WSN simulators [32] and by applying smart approaches such as context-aware sensing [33] (adjusting sensing duty cycle w.r.t. people presence in a building), ultra low power overlays [37] and on-board intelligence [38] when a sensor node implementing a sensor fusion component decides whether the measured data contain useful information, and by energy aware physical layer and medium access control design [39].

\section{CONCLUSION}

In this paper, we have demonstrated small size low power consumption gas WSN platform with thermo compensation algorithm. The platform consists of two $3 \mathrm{~cm}^{2}$ boards fitting into $5.5 \mathrm{~cm}^{3}$ packaging, which significantly improves the state-of-the-art and ensures the platform ubiquitous deployment including difficult to access areas.

To guarantee the sensor node long-term operation and stability under the changing environmental conditions, we, for the first time, presented the gas measurement procedure for the one sensor-based sensing circuit which significantly reduces its power consumption up to $1 \mathrm{~mW}$ for the periodic measurement and data transmission mode. Our experimental results have shown that the application of the thermo compensation adjustment algorithm secures the sensor zero offset stability within $\pm 1 \mathrm{mV}$, absolute measurement error within $0.1 \%$ vol. and performance. Besides, we further improved the gas WSN state-of-the art in terms of the average power consumption of the sensing circuit. The achieved results have got great potential for the industries dealing with the combustible gases detection in outdoor or harsh environments.

\section{REFERENCES}

[1] Fundamentals of Combustible Gas Detection. New York, NY, USA: General Motors, 2000.

[2] M. Magno et al., "Extended wireless monitoring through intelligent hybrid energy supply," IEEE Trans. Ind. Electron., vol. 61, no. 4, pp. 1871-1881, Apr. 2014.

[3] L. Mottola, G. P. Picco, M. Ceriotti, Ş. Gunà, and A. L. Murphy, "Not all wireless sensor networks are created equal: A comparative study on tunnels," ACM Trans. Sensor Netw. vol. 7, no. 2, article 15, 33pp., Sep. 2010.

[4] A. Somov, A. Baranov, D. Spirjakin, and R. Passerone, "Circuit design and power consumption analysis of wireless gas sensor nodes: Onesensor versus two-sensor approach," IEEE Sensors J., vol. 14, no. 6, pp. 2056-2063, Jun. 2014.

[5] J. W. Gardner, P. K. Guha, F. Udrea, and J. A. Covington, "CMOS interfacing for integrated gas sensors: A review," IEEE Sensors J., vol. 10, no. 12 , pp. $1833-1848$, Dec. 2010.

[6] C. Bambang Dwi Kuncoro et al., "Wireless e-nose sensor node: State of the art," Proc. Eng., vol. 41, pp. 1405-1411, 2012.

[7] A. Somov et al., "Deployment and evaluation of a wireless sensor network for methane leak detection," Sens. Actuators A, Phys., vol. 202, no. 11, pp. 217-225, Nov. 2013.

[8] J. Hayes, S. Beirne, K.-T. Lau, and D. Diamond, "Evaluation of a low cost wireless chemical sensor network for environmental monitoring," in Proc. IEEE Sensors, 2008, pp. 530-533.

[9] Y. W. Kim, S. J. Lee, G. H. Kim, and G. J. Jeon, "Wireless electronic nose network for realtime gas monitoring system," in Proc. IEEE Int. Workshop Robot. Sens. Environ. (ROSE'09), 2009, pp. 169-172.

[10] Electrical Apparatus for the Detection of Combustible Gases in Domestic Premises. Test Methods and Performance Requirements, EN 50194:2000, 2000.

[11] S. Ho, F. Koushanfar, A. Kosterev, and F. Tittel, "LaserSPECks: Laser spectroscopic trace-gas sensor networks-sensor integration and application," in Proc. Int. Conf. Inf. Process. Sensor Netw., 2007, pp. 226-235.

[12] A. Somov, A. Baranov, and D. Spirjakin, "A wireless sensor-actuator system for hazardous gases detection and control," Sens. Actuators A, Phys., vol. 210, pp. 157-164, Apr. 2014.

[13] K. Yokosawa, K. Saitoh, S. Nakano, Y. Goto, and K. Tsukada, "FET hydrogen-gas sensor with direct heating of catalytic metal," Sens. Actuators B, Chem., vol. 130, no. 1, pp. 94-99, Mar. 2008.

[14] A. Somov et al., "Energy-aware gas sensing using wireless sensor networks," in Proc. 9th Eur. Conf. Wireless Sensor Netw., 2012, vol. 7158, pp. 245-260.

[15] V. C. Gungor and G. P. Hancke, "Industrial wireless sensor networks: Challenges, design principles, and Technical approaches," IEEE Trans. Ind. Electron., vol. 56, no. 10, pp. 4258-4265, Oct. 2009.

[16] C. Alippi, G. Anastasi, M. Di Francesco, and M. Roveri, "Energy management in wireless sensor networks with energy-hungry sensors," IEEE Instrum. Meas. Mag., vol. 12, no. 2, pp. 16-23, Apr. 2009.

[17] N. Baccour et al., "Radio link quality estimation in wireless sensor networks: A survey," ACM Trans. Sensor Netw., vol. 8, no. 4, pp. 1-35, Nov. 2012.

[18] O. Kreibich, J. Neuzil, and R. Smid, "Quality-based multiple-sensor fusion in an industrial wireless sensor network for MCM," IEEE Trans. Ind. Electron., vol. 61, no. 9, pp. 4903-4911, Sep. 2014.

[19] G. Korotcenkov and B. K. Cho, "Engineering approaches to improvement of conductometric gas sensor parameters. Part 2: Decrease of dissipated (consumable) power and improvement stability and reliability," Sens. Actuators B, Chem., vol. 198, pp. 316-341, 2014.

[20] Requirements for Setting Up Stationary Gas Analysers at Industrial Premises and Outdoor Areas of Oil and Gas Enterprises, Standard RD BT 39-0147171-003-88, 2009.

[21] OpenPicus. FlyPort Sensing Platform [Online]. Available: http://www. openpicus.com

[22] Libelium. WaspMote Sensing Platform [Online]. Available: http://www. libelium.com/products/waspmote/

[23] P. Vlacheas et al., "Enabling smart cities through a cognitive management framework for the internet of things," IEEE Commun. Mag., vol. 51, no. 6 , pp. 102-111, Jun. 2013. 
[24] A. Somov et al., "Development of wireless sensor network for combustible gas monitoring," Sens. Actuators A, Phys., vol. 171, no. 2, pp. 398-405, Jul. 2011.

[25] P. Cheong et al., "A zigbee-based wireless sensor network node for ultraviolet detection of flame," IEEE Trans. Ind. Electron., vol. 58, no. 11, pp. 5271-5277, Nov. 2011.

[26] Y. H. Chee et al., "PicoCube: A $1 \mathrm{~cm}^{3}$ sensor node powered by harvested energy," in Proc. 45th ACM/IEEE Des. Autom. Conf. (DAC'08), 2008, pp. 114-119.

[27] B. Zhang, R. Simon, and H. Aydin, "Harvesting-aware energy management for time-critical wireless sensor networks with joint voltage and modulation scaling," IEEE Trans. Ind. Informat., vol. 9, no. 1, pp. 514-526, Feb. 2013.

[28] J. Lu, H. Okada, T. Itoh, T. Harada, and R. Maeda, "Towards the world smallest wireless sensor nodes with ultra-low power consumption," IEEE Sensors J., vol. 14, no. 6, pp. 2035-2041, Jun. 2014.

[29] E. E. Karpov et al., "Energy efficient planar catalytic sensor for methane measurement," Sens. Actuators A, Phys., vol. 194, no. 1, pp. 176-180, May 2013.

[30] S. Okazaki, H. Nakagawa, S. Asakura, H. Shimizu, and I. Iwamoto, "A novel method of temperature compensation for a stable combustion-type gas sensor," Sens. Actuators B, Chem., vol. 77, pp. 322-325, 2001.

[31] H. Ma, E. Ding, and W. Wang, "Power reduction with enhanced sensitivity for pellistor methane sensor by improved thermal insulation packaging," Sens. Actuators B, Chem., vol. 187, pp. 221-226, Oct. 2013.

[32] I. Minakov and R. Passerone, "PASES: An energy-aware design space exploration framework for wireless sensor networks," Syst. Arch., vol. 59, no. 8, pp. 626-642, Sep. 2013.

[33] V. Jelicic, M. Magno, D. Brunelli, G. Paci, and L. Benini, "Contextadaptive multimodal wireless sensor network for energy-efficient gas monitoring," IEEE Sensors J., vol. 13, no. 1, pp. 328-338, Jan. 2013.

[34] D. Wobscholl, "A wireless gas monitor with IEEE 1451 protocol," in Proc. IEEE Sensors Appl. Symp., 2006, pp. 162-164.

[35] I. Sayhan et al., "Discontinuously operated metal oxide gas sensors for flexible tag microlab applications," IEEE Sensors J., vol. 8, no. 2, pp. 176-181, Feb. 2008

[36] S. Bicelli et al., "Model and experimental characterization of the dynamic behavior of low-power carbon monoxide MOX sensors operated with pulsed temperature profiles," IEEE Trans. Instrum. Meas., vol. 58, no. 5, pp. 1324-1332, May 2009

[37] M. Magno, D. Boyle, D. Brunelli, E. Popovici, and L. Benini, "Ensuring survivability of resource-intensive sensor networks through ultra-low power overlays," IEEE Trans. Ind. Informat., vol. 10, no. 2, pp. 946-956, May 2014.

[38] S. De Vito et al., "Wireless sensor networks for distributed chemical sensing: Addressing power consumption limits with on-board intelligence," IEEE Sensors J., vol. 11, no. 4, pp. 947-955, Apr. 2011.

[39] T. Kim, H. Kim II, Y. Sun, and Z. Y. Jin, "physical layer and medium access control design in energy efficient sensor networks: An overview," IEEE Trans. Ind. Informat., vol. 11, no. 1, pp. 2-15, Feb. 2015.

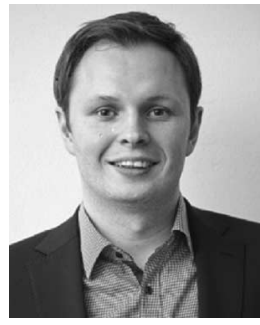

Andrey Somov (M'14) graduated in 2004 and received the Diploma in electronics engineering in 2006 from MATI, Russian State Technological University, Russia, and received the Ph.D. degree in power management in wireless sensor networks from the University of Trento, Trento, Italy, in 2009.

$\mathrm{He}$ is a Senior Researcher in the area of smart Internet of Things at the CREATE-NET Research Center, Trento. Before starting his Ph.D., he worked as an Electronics Engineer in space technology with VNIIEM Corporation, Moscow, Russia. In the fall 2008, he was a Visiting Researcher at the University of California, Berkeley, CA, USA, where he conducted research on energy-efficient sensor networks. He has authored more than 30 papers in peer-reviewed international journals and conferences. His research interests include power management for the wireless sensor nodes, cognitive Internet of Things, and associated proof-of-concept implementation.

Dr. Somov has been the General Chair of the 6th International Conference on Sensor Systems and Software (S-Cube'15) and the "IoT360" Summer School on the Internet of Things in 2014 and 2015.

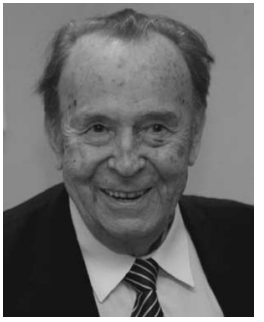

Evgeny F. Karpov received the Ph.D. degree in technical science from Moscow State Mining University, USSR, in 1961.

$\mathrm{He}$ is a Scientific Consultant with the Scientific Technical Centre of Gas Measuring Sensors, Lyubertsy, Russia. He acted as a Project Leader on several national and international research projects in life safety technology. He is the author of more than 200 scientific publications, including seven books and more than 70 patents in gas measuring instruments.

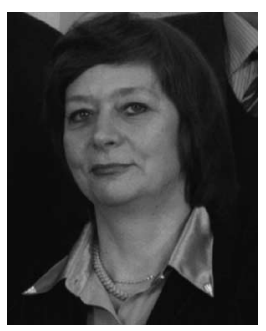

Elena Karpova received the Diploma of Physicist and $\mathrm{Ph} . \mathrm{D}$. degrees in physics and mathematics from Moscow State University, Moscow, Russia, in 1984.

She is an Associate Professor with STANKIN, Moscow State Technological University, Moscow, Russia. Her research interests include aspects of diffusion and thermoconductivity in gas analysis.

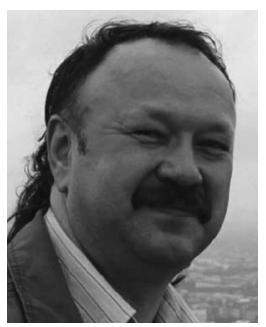

Alexey Suchkov (Deceased) received the Ph.D degree in life safety technology from the Academy of Science Mining Engineering Institute, Russia, in 2003.

He was a Chief Engineer with the Scientific Technical Centre of Gas Measuring Sensors, Lyubertsy, Russia.

Sergey Mironov received the Diploma degree in electrical engineering from Moscow Power Engineering Institute, Moscow, USSR, in 1979.

$\mathrm{He}$ is an Engineer with the Scientific Technical Centre of Gas Measuring Sensors (NTC IGD), Lyubertsy, Russia. His research interests include the development of two-dimensional catalytic sensors and dynamic measuring methods.

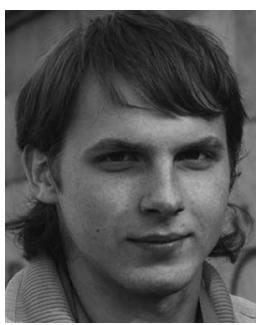

Alexey Karelin received the B.S. and M.S. degrees in electronic engineering from MATI, Russian State Technological University, Moscow, Russia, in 2005 and 2007, respectively. He is currently pursuing the $\mathrm{Ph} . \mathrm{D}$. degree at the same university.

His research interests include the detection of flammable gas mixtures, investigation of planar catalytic sensors, and development of embedded systems.

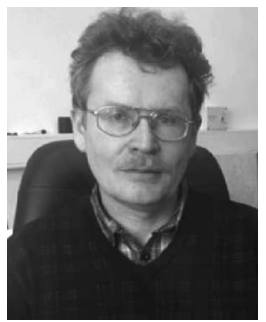

Alexander Baranov (M'15) graduated from the Moscow Institute of Electronic Machinery, Moscow, Russia, in 1987. He received the Ph.D. degree in physics and mathematics from the Moscow Institute of Electronics and Mathematics Technical University, Moscow, and the Doctor of Technical Science degree from MATI, Russian State Technological University, Moscow, in 1994 and 2003, respectively.

$\mathrm{He}$ is a Professor with MATI, Russian State Technological University. He is the Project Leader on several national and international research projects. His research interests include the energy efficient wireless gas sensors, gas sensors development and characterization, wireless sensor networks, energy harvesting technology for wireless application, and thin film nanocomposite catalysts. 


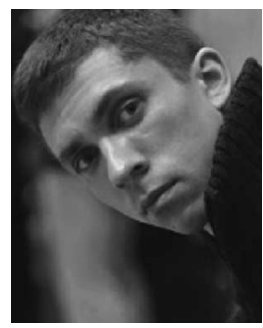

Denis Spirjakin received the B.S. and M.S. degrees in electronic engineering from MATI, Russian State Technological University, Moscow, Russia, in 2005 and 2007, respectively. He is currently pursuing the $\mathrm{Ph} . \mathrm{D}$. degree at the same university.

His research interests include digital signal processing, data transmission systems, and wired/ wireless sensor networks. 